\title{
Multifractal variability in geoelectrical signals and correlations with seismicity: a study case in southern Italy
}

\author{
L. Telesca, G. Colangelo, and V. Lapenna \\ Institute of Methodologies for Environmental Analysis, CNR, C. da S. Loja, 85050 Tito (PZ), Italy \\ Received: 17 May 2005 - Revised: 29 July 2005 - Accepted: 29 July 2005 - Published: 9 September 2005 \\ Part of Special Issue "Seismic hazard evaluation, precursory phenomena and seismo electromagnetics"
}

\begin{abstract}
Multifractal fluctuations in the time dynamics of geoelectrical data, recorded in a seismic area of southern Italy, have been revealed using the Multifractal Detrended Fluctuation Analysis (MF-DFA), which allows to detect multifractality in nonstationary signals. Our findings show that the geoelectrical time series, recorded in the seismic area of southern Apennine Chain (Italy), is multifractal. The time evolution of the multifractality suggests that the multifractal degree increases prior the occurrence of earthquakes. This study aims to propose another approach to investigate the complex dynamics of earthquake-related geoelectrical signals.
\end{abstract}

\section{Introduction}

Scientific research focused on the complexity of earthquakes and earthquake-related geophysical variability has evidenced a growing interest in recent years. The monitoring of time variability of geophysical fields may be useful to gain information on phenomena linked to seismic activity (i.e. Rikitake, 1988; Zhao et al., 1994; Park, 1997; Martinelli and Albarello, 1997; Di Bello et al., 1998; Vallianatos and Tzanis, 1999; Hayakawa et al., 2000; Telesca et al., 2001; Tramutoli et al., 2001). In particular, variations in the stress and fluid flow fields can produce changes in the geoelectrical signals (Scholz, 1990), which can be used to obtain information on the governing mechanisms both in normal conditions and during intense seismic activity.

Geoelectrical signals are voltage difference between two points on Earth's surface due to the presence of an electric field produced by natural sources distributed in the subsoil (e.g. Parasnis, 1986; Sharma, 1997 and references therein). The most relevant phenomenon that could originate the geoelectrical field is known as streaming potential: the electrical signal is produced when a fluid flows in a porous rock due

Correspondence to: L. Telesca

(ltelesca@imaa.cnr.it) to a pore pressure gradient. The phenomenon is generated by the formation within the porous ducts of a double electrical layer between the bounds of the solid, that absorbs electrolytic anions and cations distributed in a diffused layer near the boards. The dissolved salts increase the amount of anions and cations of the underground liquids. The free liquid in the centre of the rock pore is usually enriched in cations, while anions are usually absorbed on the soil surface in silicate rock. The free pore water carries an excess positive charge, a part of which accumulates close to the solid-liquid interface forming a stable double layer. When the liquid is forced through the porous medium, the water molecules carry free positive ions in the diffusion part of the pore. This relative movement of cations with reference to the firmly attached anions generates the well know streaming potential (Keller and Frischknecht, 1966), which, as suggested by Mizutani et al. (1976), can be responsible for the voltage measures on the ground surface preceding an earthquake (Patella, 1997). In a seismic focal region the effect could be enhanced due to increasing accumulation of strain, which can cause dilatancy of rocks (Nur, 1972).

Geoelectrical signals could reflect the irregularity and heterogeneity of the crust. Therefore the structure of the geoelectrical signal could be linked to the structure of the seismic focal zone. In fact, the geometry and the structure of individual fault zones can be represented by a network with an anisotropic distribution of fracture orientations, and consisting of fault-related structures including small faults, fractures, veins and folds. This is a consequence of the roughness of the boundaries between each component and the interaction between the distinct components within the fault zone (O'Brien et al., 2003). In fact earthquake faulting is characterized by irregular rupture propagation and non-uniform distributions of rupture velocity, stress drop and co-seismic slip. These observations indicate a non-uniform distribution of strength in the fault zone, whose geometry and mechanical heterogeneities are important factors to be considered in the prediction of strong motion. Experimental studies on the hierarchical nature of the processes underlying fault rupture, 


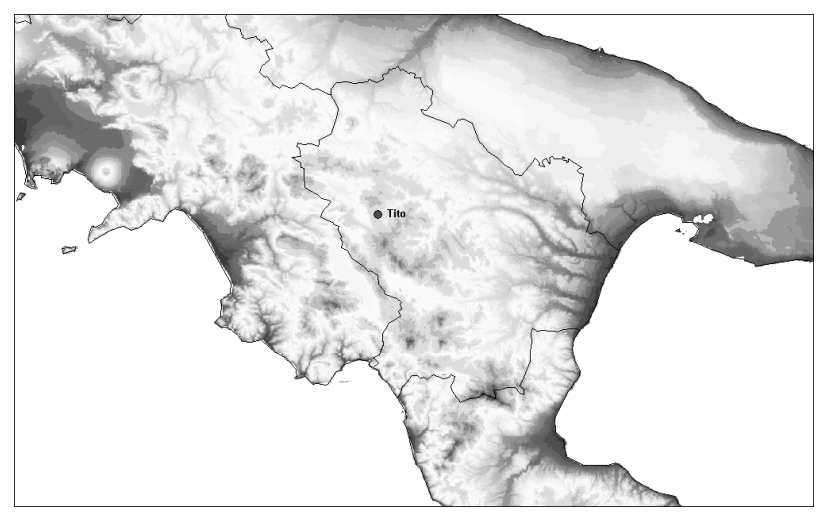

Fig. 1. Location of the geoelectrical station Tito in southern Italy.

leading to the possibility of recognizing the final preparation stage before a large earthquake have been performed (Lei et al., 2003). Cowie et al. (1993) have introduced a numerical rupture model to simulate the growth of faults in a tectonic plate driven by a constant plate boundary velocity. They found that the plate initially deforms by uncorrelated nucleation of small faults reflecting the distribution of material properties. Due to increase of strain, growth and coalescence of existing faults dominate over nucleation and a power-law distribution of fault size appears, characterizing the fault pattern as a fractal. Cowie et al. (1995) have shown that the combined effect of fault clustering and the correlation between fault displacement and fault size leads to a strongly multifractal deformation pattern.

To quantitatively characterize geoelectrical dynamics, techniques able to extract robust features hidden in their complex fluctuations are needed. Fractality is one of the features of such complexity. What does fractality mean? A fractal is an object whose sample path included within some radius scales with the size of the radius. It is clear from the definition of fractal, that fractal processes are characterized by scaling behaviour, which leads naturally to power-law statistics. In fact, consider a statistics $f(x)$, which depends continuously on the scale $x$, over which the measurements are taken. Suppose that changing the scale $x$ by a factor $a$, will effectively scale the statistics $f(x)$ by another factor $g(a), f(a x)=g(a) f(x)$. The only nontrivial solution for this scaling equation is given by $f(x)=b g(x), g(x)=x^{c}$, for some constants $b$ and $c$ (Thurner et al., 1997 and references therein). Therefore, power-law statistics and fractals are very closely related concepts.

The fractality of a signal can be investigated aiming to characterize its temporal fluctuations; in this case, we need to perform second-order fractal measures, which furnish information regarding the correlation properties of a time series. The spectral analysis represented the standard method to detect correlation features in time series fluctuations. The power spectrum is obtained by means of the Fourier Transform of the signal. It describes how the power is concentrated at various frequency bands. Thus, the power spec- trum reveals periodic, multiperiodic or non-periodic signals. The fractality of a time series is revealed by a power-law dependence of the spectrum upon the frequency, $S(f) \sim 1 / f^{\alpha}$, where the scaling (spectral) exponent $\alpha$ informs on the type and the strength of the time-correlation structures intrinsic in the signal fluctuations (Havlin et al., 1999). If $\alpha=0$ the temporal fluctuations are purely random, typical of white noise processes, characterized by completely uncorrelated samples. If $\alpha>0$, the temporal fluctuations are persistent, meaning that positive (negative) variations of the signal will be very likely followed by positive (negative) variations; this feature is typical of system which are governed by positive feedback mechanisms. If $\alpha<0$, the temporal fluctuations are antipersistent, meaning that positive (negative) variations of the signal will be very likely followed by negative (positive) variations; this feature is typical of system which are governed by negative feedback mechanisms.

The estimate of the spectral exponent is rather rough, due to large fluctuations in the power spectrum, especially at high frequencies. Furthermore, the power spectrum is sensitive to nonstationarities that could be present in observational data.

For this reason, different fractal methods, as the Higuchi method or the detrended fluctuation analysis, have been developed to furnish stable estimates of the spectral exponent (Higuchi, 1988, 1990), or to allow the detecting of scaling behaviours in experimental time series, very often affected by trends and nonstationarities, which cause spurious detection of correlations (Peng et al., 1995).

But all these techniques are monofractal, and very often they are not sufficient to describe the scaling properties of a signal, which could be multifractal.

What does multifractality mean? A multifractal is an object which needs many exponents to characterize its scaling properties. It can be decomposed into many sub-sets characterized by different scaling exponents. Thus multifractals are intrinsically more complex and inhomogeneous than monofractals, and characterize systems featured by irregular dynamics, with sudden bursts of high frequency fluctuations.

The aim of the present paper is the dynamical investigation of a geoelectrical time series recorded in southern Italy, one of the most seismically active area of the Mediterranean Region. Our purpose is to characterize the multifractality of such time series in order to reveal a possible correlations with the seismic activity of the area.

\section{Data}

We study a geoelectrical data set recorded at Tito station $\left(40.602^{\circ} \mathrm{N}, 15.724^{\circ} \mathrm{E}\right)$, located in one of the most seismically active areas of southern Italy (Fig. 1). The signal consists of voltage difference between two no polarizable electrodes inserted vertically in the ground to avoid the external meteo-climatic effects. The two dipoles are placed at $15 \mathrm{~m}$ and $20 \mathrm{~m}$, respectively. Figure 2 shows the time series, which consists of minute-sampled geoelectrical values, recorded from 1 July 2004 and 30 November 2004. There are some 
data missings due to drawbacks in the technical equipment. There are also shown the earthquakes occurred during the observation period and satisfying Dobrovolskiy's rule (Dobrovolskiy et al., 1979; Dobrovolskiy, 1993). This law, which is a theoretical relation between earthquake magnitude, distance from the epicenter and volumetric strain, states that detectable seismically induced strain exceeds $10^{-8}$. From this relation the maximum distance from the epicenter in which the effects of the earthquake are detectable is $r=10^{0.43 M}$, where $r$ is measured in $\mathrm{km}$.

\section{Methods and data analysis}

Observational data often present clear irregular dynamics, characterized by sudden bursts of high frequency fluctuations, which suggest performing a multifractal analysis evidencing the presence of different scaling behaviours for different intensities of fluctuations. Furthermore, the signal may appear nonstationary.

The Multifractal Detrended Fluctuation Analysis (MFDFA) (Kantelhardt et al., 2002) is a useful tool to characterize multifractality in nonstationary data.

The method is based on the conventional detrended fluctuation analysis (Peng et al., 1995). It operates on the time series $x(i)$, where $i=1,2, \ldots, N$ and $N$ is the length of the series. With $x_{\text {ave }}$ the mean value is indicated. Assume that $x(i)$ are increments of a random walk process around the average $x_{\text {ave }}$, the "trajectory" or "profile" is given by the integration of the signal

$y(i)=\sum_{k=1}^{i}\left[x(k)-x_{\mathrm{ave}}\right]$.

Next, the integrated time series is divided into $N_{S}=\int(N / s)$ no overlapping segments of equal length $s$. Since the length $N$ of the series is often not a multiple of the considered time scale $s$, a short part at the end of the profile $y(i)$ may remain. In order not to disregard this part of the series, the same procedure is repeated starting from the opposite end. Thereby, $2 N_{S}$ segments are obtained altogether. Then the local trend for each of the $2 N_{S}$ segments is calculated by a least square fit of the series. Then one calculates the variance

$F^{2}(s, v)=\frac{1}{s} \sum_{i=1}^{s}\left\{y[(v-1) s+i]-y_{v}(i)\right\}^{2}$

for each segment $v, \nu=1, \ldots, N_{S}$ and

$F^{2}(s, v)=\frac{1}{s} \sum_{i=1}^{s}\left\{y\left[N-\left(v-N_{S}\right) s+i\right]-y_{v}(i)\right\}^{2}$

for $v=N_{S}+1, \ldots, 2 N_{S}$. Here, $y_{v}(i)$ is the fitting line in segment $v$. Then, an average over all segments is performed to obtain the $q-t h$ order fluctuation function

$F_{q}(s)=\left\{\frac{1}{2 N_{S}} \sum_{\nu=1}^{2 N_{S}}\left[F^{2}(s, v)\right]^{\frac{q}{2}}\right\}^{\frac{1}{q}}$

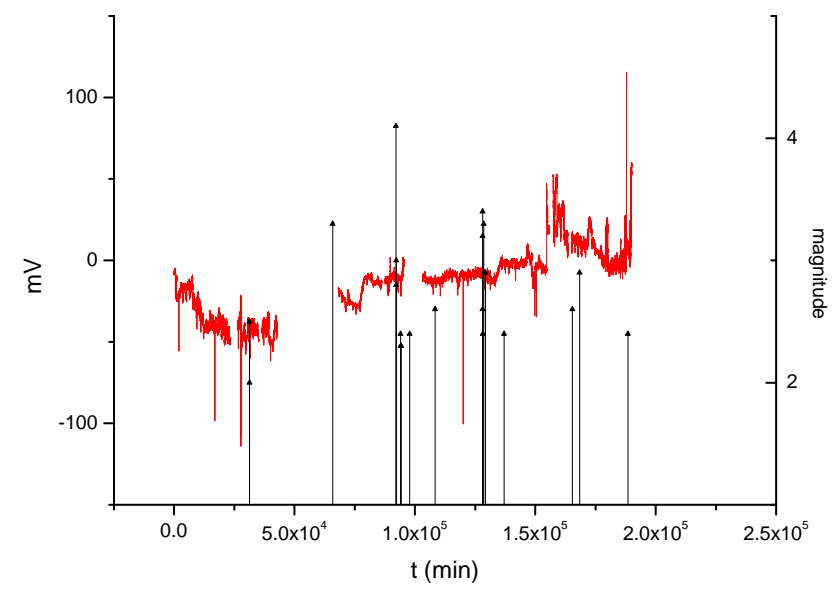

Fig. 2. Time variation of the geoelectrical signal, measured at Tito site, and the earthquakes (vertical arrows) occurred in the area and satisfying the Dobrovolskiy's rule. This rule has permitted the selection of 21 earthquakes. The data missings are due to drawbacks in the technical equipment.

where, in general, the index variable $q$ can take any real value except zero.

Repeating the procedure described above, for several time scales $s, F_{q}(s)$ will increase with increasing $s$. Then analyzing $\log -\log$ plots $F_{q}(s)$ versus $s$ for each value of $q$, the scaling behaviour of the fluctuation functions can be determined. If the series $x_{i}$ is long-range power-law correlated, $F_{q}(s)$ increases for large values of $s$ as a power-law

$F_{q}(s) \propto s^{h(q)}$.

The value $h(0)$ corresponds to the limit $h(q)$ for $q \rightarrow 0$, and cannot be determined directly using the averaging procedure of Eq. (4) because of the diverging exponent. Instead, a logarithmic averaging procedure has to be employed,

$F_{0}(s) \equiv \exp \left\{\frac{1}{4 N_{S}} \sum_{\nu=1}^{2 N_{S}} \ln \left[F^{2}(s, v)\right]\right\} \approx s^{h(0)}$.

In general the exponent $h(q)$ will depend on $q$. For stationary time series, $h(2)$ is the well-defined Hurts exponent $H$ (Feder, 1988). Thus, we call $h(q)$ the generalized Hurst exponent. Monofractal time series are characterized by $h(q)$ independent of $q$. The different scaling of small and large fluctuations will yield a significant dependence of $h(q)$ on $q$. For positive $q$, the segments $v$ with large variance (i.e. large deviation from the corresponding fit) will dominate the average $F q(s)$. Therefore, if $q$ is positive, $h(q)$ describes the scaling behaviour of the segments with large fluctuations; and generally, large fluctuations are characterized by a smaller scaling exponent $h(q)$ for multifractal time series. For negative $q$, the segments $v$ with small variance will dominate the average $F q(s)$. Thus, for negative $q$ values, the scaling exponent $h(q)$ describes the scaling behaviour of segments with small fluctuations, usually characterized by larger scaling exponents. 


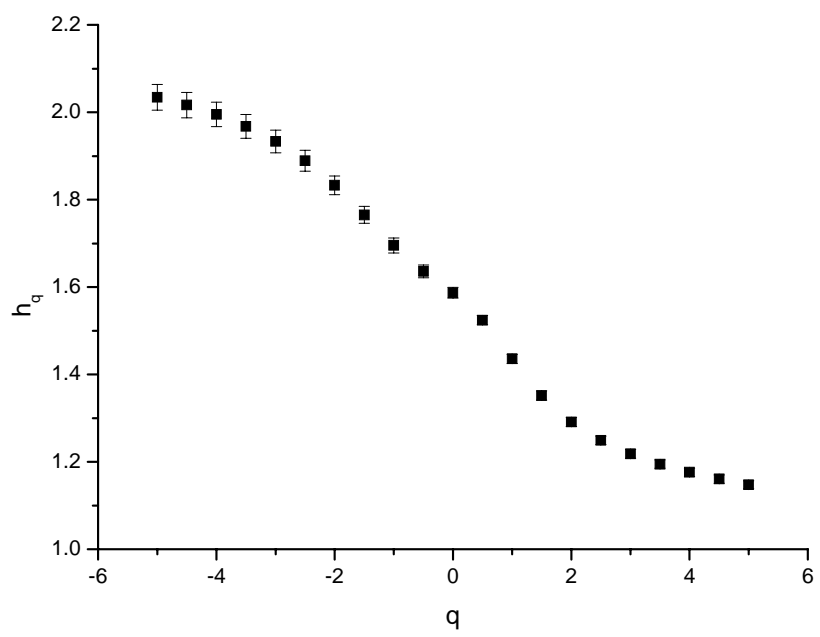

Fig. 3. $h(q) \sim q$ relation for the geoelectrical Tito time series.

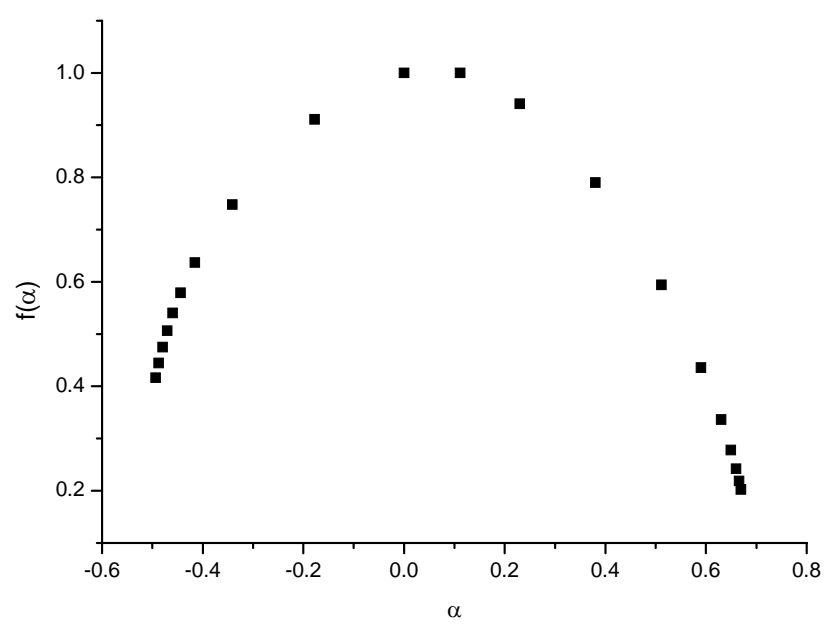

Fig. 4. Singularity spectra of the geoelectrical Tito time series.

Figure 3 shows the $q$-dependence of the generalized Hurst exponent $h(q)$ determined by fits in the regime $10^{2} \min <s<N / 4$, where $N$ indicates the length of the series and for $q$ ranging between -5 and 5 with 0.5 step.

The multifractal scaling exponents $h(q)$ are directly related to the scaling exponents $\tau(q)$ defined by the standard partition function multifractal formalism (Kantelhardt et al., 2002)

$\tau(q)=q h(q)-1$.

The singularity spectrum $f(\alpha)$ is related to $\tau(q)$ by means of the Legendre transform (Parisi and Frish, 1985),

$\alpha=\frac{d \tau}{d q}$

$f(\alpha)=q \alpha-\tau(q)$,

where $\alpha$ is the Hölder exponent and $f(\alpha)$ indicates the dimension of the subset of the series that is characterized by $\alpha$.

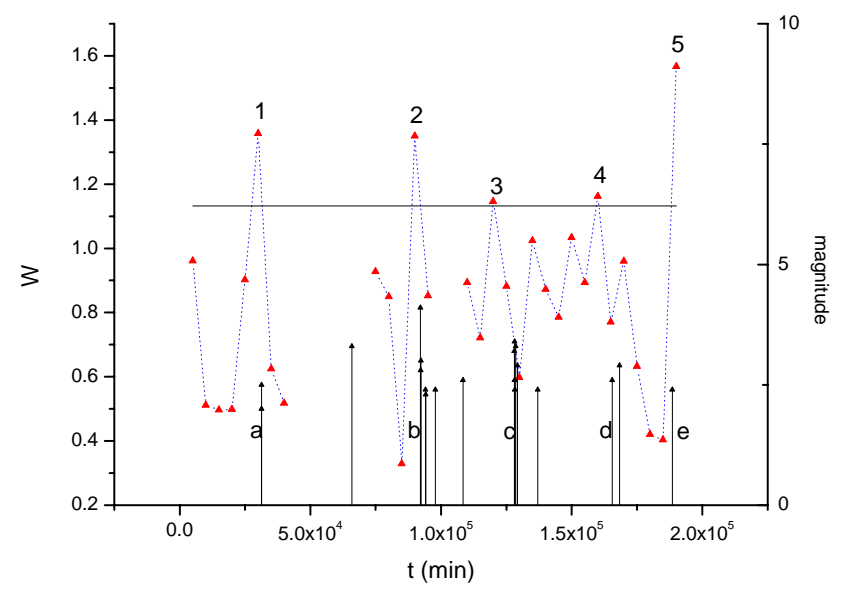

Fig. 5. Time variation of the $\alpha$-range for the data plotted in Fig. 2 . The horizontal line indicates the value of the standard deviation of all the $W$ values. The values of $W$ above this threshold are correlated with the earthquakes. The letters a-e indicates the single earthquakes or the seismic clusters, which can be put in connection with the increase of $W$.

The singularity spectrum quantifies in details the long-range correlation properties of a time series. Figure 4 shows the multifractal spectrum $f(\alpha)$ for the signal.

We investigated the time variation of the multifractal behaviour of the series in order to find possible correlation with the earthquakes occurred in the area during the observation period. We have calculated the set of the generalized Hurst exponent $\left\{h_{q}(t):-5 \leq q \leq+5\right\}$ in no-overlapping time windows $5 \times 10^{3} \mathrm{~min}$ long. Then we calculated the difference between the maximum and minimum $\alpha$-value ( $\alpha$-range) in the multifractal spectra. The $\alpha$-range can be considered as a measure of the width of the multifractal spectrum, which indicates the degree of multifractality. Figure 5 shows the $\alpha$ range varying with time: clear enhancement of the $\alpha$-range (above the threshold, given by the standard deviation and indicated by the horizontal line in the plot) is visible prior the occurrence of earthquakes or seismic clusters (indicated in Fig. 5 with the letters a-e).

\section{Conclusions}

The geophysical phenomenon underlying the self-potential variability connected to earthquake activity is complex and is governed by physical laws that are not completely known. The multifractal analysis has led to a better understanding of such complexity, by means of the generalized Hurst exponents and the singularity spectrum. The singularity spectrum has led to a better description of the signal revealing a clear enhancement of its multifractal degree (measured by the variation of the $\alpha$-range) prior the occurrence of the earthquakes, selected by Dobrovolskiy's rule. Of course, in order to be able to assess significant correlations between earthquakes and patterns of multifractal parameters, and, in particular, the use of such patterns to perform feasible 
earthquake prediction, we need to investigate data set longer than that examined in this paper and measured in different seismotectonic environments. Nevertheless, the use of multifractal tools can be promising aiming to better characterize the time dynamics of earthquake-related geophysical phenomena.

Edited by: P. F. Biagi

Reviewed by: three referees

\section{References}

Cowie, P. A., Vanneste, C., and Sornette, D.: Statistical physics model for the spatio-temporal evolution of faults, J. Geophys. Res., 98, 21 809-21 821, 1993.

Cowie, P. A., Sornette, D., and Vanneste, C.: Multifractal scaling properties of a growing fault population, Geophys. J. Int., 122, 457-469, 1995.

Di Bello, G., Heinicke, J., Koch, U., Lapenna, V., Macchiato, M., Martinelli, G., and Piscitelli, S.: Geophysical and geochemical parameters jointly monitored in a seismic area of Southern Apennines (Italy), Phys. Chem. Earth, 23, 909-914, 1998.

Dobrovolsky, I. P.: Analysis of preparation of a strong tectonic earthquake, Phys. Solid Earth, 28, 481-492, 1993.

Dobrovolsky, I. P., Zubkov, S. I., and Miachkin, V. I.: Estimation of the size of earthquake preparation zones, Pageoph, 117, 10251044, 1979.

Kantelhardt, J. W., Zschiegner, S. A., Koscielny-Bunde, E., Havlin, S., Bunde, A., and Stanley, H. E.: Multifractal detrended fluctuation analysis of nonstationary time series, Physica A, 316, 87114,2002

Keller, G. V. and Frischknecht, F. C.: Electrical Methods in Geophysical Prospecting, Pergamon Press, Oxford, 523 pp., 1966.

Havlin, S., Amaral, L. A. N., Ashkenazy, Y., Goldberger, A. L., Ivanov, P. Ch. Peng, C.-K., and Stanley, H. E.: Application of statistical physics to heartbeat diagnosis, Physica A, 274, 99110, 1999.

Hayakawa, M., Hattori, K., Itoh, T., and Yumoto, K.: ULF electromagnetic precursors for an earthquake at Biak, Indonesia on 17 February 1996, Geophys. Res. Lett., 27, 1531-1534, 2000.

Higuchi, T.: Approach to an irregular time series on the basis of the fractal theory, Physica D, 31, 277-283, 1988.

Higuchi, T.: Relationship between the fractal dimension and the power law index for a time series: a numerical investigation, Physica D, 46, 254-264, 1990.

Feder, J.: Fractals, Plenum Press, New York, 283 pp., 1988.

Lei, X., Kusunose, K., Nishizawa, O., and Satoh, T.: The hierarchical rupture process of a fault: an experimental study, Phys. Earth Planet. Int. 137, 213-228, 2003.
Martinelli, G. and Albarello, D.: Main constraints for siting monitoring networks devoted to the study of earthquake related hydrogeochemical phenomena in Italy, Annali di Geofisica, 40, 15051522, 1997.

Mizutani, H., Ishido, T., Yokokura, T., and Ohnishi, S.: Electrokinetic phenomena associated with earthquakes, Geophys. Res. Lett., 3, 365-368, 1976.

Nur, A.: Dilatancy pore fluids and premonitory variations of $t s / t p$ travel times, Bull. Seism. Soc. Am., 62, 1217-1222, 1972.

O'Brien, G. S., Bean, C. J., and McDermott, F.: A numerical study of passive transport through fault zones, Earth Planet. Sci. Lett., 214, 633-643, 2003.

Parasnis, D. S.: Principles of Applied Geophysics, Chapman and Hall, 402 pp., 1986.

Parisi, G. and Frisch, U.: In: Turbulence and Predictability in Geophysical Fluid Dynamics and Climate Dynamics, edited by: Ghil, M., Benzi, R., and Parisi, G., North Holland, Amsterdam, 84-87, 1985.

Park, S. K.: Monitoring resistivity changes in Parkfield, California 1988-1995, J. Geophy. Res., 102, 24 545-24 559, 1997.

Patella, D.: Introduction to ground surface SP tomography, Geophys. Prospect., 45, 653-681, 1997.

Peng, C.-K., Havlin, S., Stanley, H. E., and Goldberger, A. L.: Quantification of scaling exponents and crossover phenomena in nonstationary heartbeat time series, Chaos, 5, 82-87, 1995.

Rikitake, T.: Earthquake prediction: an empirical approach, Tectonophysics, 148, 195-210, 1988.

Scholz, C. H.: The mechanics of earthquakes and faulting, Cambridge University Press, 439 pp., 1990.

Sharma, P. S.: Enviromental and Engineering Geophysics, Cambridge Univ. Press, 499 pp., 1997.

Telesca, L., Cuomo, V., Lapenna, V., and Macchiato, M.: A new approach to investigate the correlation between geoelectrical time fluctuations and earthquakes in a seismic area of southern Italy, Geophys. Res. Lett., 28, 4375-4378, 2001.

Thurner, S., Lowen, S. B., Feurstein, M. C., Heneghan, C., Feichtinger, H. C., and Teich, M. C.: Analysis, Synthesis, and Estimation of Fractal-Rate Stochastic Point Processes, Fractals, 5, 565-596, 1997.

Tramutoli, V., Di Bello, G., Pergola, N., and Piscitelli, S.: Robust satellite techniques for remote sensing of seismically active areas, Annali di Geofisica, 44, 295-312, 2001.

Vallianatos, F. and Tzanis, A.: On possible scaling laws between Electric Earthquake Precursors (EEP) and Earthquake Magnitude, Geophys. Res. Lett., 26, 2013-2016, 1999.

Zhao, Y. and Qian, F.: Geoelectric precursors to strong earthquakes in China, Tectonophysics, 233, 99-113, 1994. 